\title{
Extensively drug-resistant Alcaligenes faecalis infection
}

\author{
Chienhsiu Huang(1)
}

\begin{abstract}
Background: Alcaligenes faecalis is usually causes opportunistic infections in humans. Alcaligenes faecalis infection is often difficult to treat due to its increased resistance to several antibiotics. The results from a clinical study of patients with Alcaligenes faecalis infection may help improve patients' clinical care.

Methods: We conducted a retrospective analysis of all patients presenting with Alcaligenes faecalis infection from January 2014 to December 2019. The medical records of all patients were reviewed for demographic information, clinical symptoms and signs, comorbidities, use of intravenous antibiotics within the past three months, bacterial culture, antibiotics sensitivity test, and clinical outcomes.

Results: Sixty-one cases of Alcaligenes faecalis infection were seen during the study period, including 25 cases of cystitis, nine cases of diabetic foot infection, eight cases of pneumonia, seven cases of acute pyelonephritis, three cases of bacteremia, and nine cases of infection at specific sites. Thirty-seven patients (60.7\%) had a history of receiving intravenous antibiotics within three months of the diagnosis. Fifty-one (83.6\%) cases were mixed with other bacterial infections. Extensively drug-resistant infections have been reported since 2018. The best sensitivity rate to Alcaligenes faecalis was $66.7 \%$ for three antibiotics (imipenem, meropenem, and ceftazidime) in 2019. Two antibiotics (ciprofloxacin and piperacillin/tazobactam) sensitivity rates to $A$. faecalis were less than $50 \%$.
\end{abstract}

Conclusions: The most frequent Alcaligenes faecalis infection sites, in order, are the bloodstream, urinary tract, skin and soft tissue, and middle ear. The susceptibility rate of Alcaligenes faecalis to commonly used antibiotics is decreasing. Extensively drug-resistant Alcaligenes faecalis infections have emerged.

Keywords: Alcaligenes faecalis infection, Extensively drug-resistant

\section{Introduction}

Alcaligenes faecalis (A. faecalis) is a Gram-negative, obligate aerobic, oxidase-positive, catalase-positive, and nonfermenting bacterium. It is commonly found in soil, water, and in hospital settings, such as in respirators, hemodialysis systems, and intravenous solutions $[1,2]$. It is a potentially emerging pathogen and usually causes opportunistic infections in humans. The organism has been isolated from a range of clinical materials, such as urine, blood, wound discharge, stool, cerebrospinal fluid,

Correspondence: hgssport@yahoo.com.tw; dm550671@tzuchi.com.tw Department of Internal Medicine, Division of Chest Medicine, Dalin Tzu Chi Hospital, Buddhist Tzu Chi Medical Foundation, NO. 2, Min-Sheng Road. Dalin Town, Chiayi County, Taiwan and respiratory secretions [3-6]. A. faecalis has been associated with endocarditis, bacteremia, meningitis, endophthalmitis, skin and soft tissue infections, urinary tract infections, otitis media, peritonitis, and pneumonia $[1,2,7-28]$. A. faecalis infection is often difficult to treat due to its increased resistance to several antibiotics, such as anti-pseudomonas penicillin, cephalosporins, carbapenems, aminoglycosides, and quinolones $[17,23,24$, 26]. Optimal antibiotic therapy for $A$. faecalis has not been well established in the literature. We report the results of a clinical study of patients with A. faecalis infection in this article. This study aims to emphasize the emergence of extensively drug-resistant $A$. faecalis and to provide local susceptibility data for the same.

C The Author(s). 2020 Open Access This article is licensed under a Creative Commons Attribution 4.0 International License, which permits use, sharing, adaptation, distribution and reproduction in any medium or format, as long as you give appropriate credit to the original author(s) and the source, provide a link to the Creative Commons licence, and indicate if changes were made. The images or other third party material in this article are included in the article's Creative Commons licence, unless indicated otherwise in a credit line to the material. If material is not included in the article's Creative Commons licence and your intended use is not permitted by statutory regulation or exceeds the permitted use, you will need to obtain permission directly from the copyright holder. To view a copy of this licence, visit http://creativecommons.org/licenses/by/4.0/ The Creative Commons Public Domain Dedication waiver (http://creativecommons.org/publicdomain/zero/1.0/) applies to the data made available in this article, unless otherwise stated in a credit line to the data. 


\section{Methods}

\section{Study design}

We conducted a retrospective analysis of all patients presenting with $A$. faecalis infection who were admitted to Dalin Tzu Chi Hospital from January 2014 to December 2019. Patients were diagnosed with A. faecalis infection when their clinical symptoms and signs indicated infection.

\section{Data collection}

The data were obtained from the hospital's clinical information system, microbiology laboratory report system, and medical chart review. We obtained details on all $A$. faecalis infections (including community-onset infection and hospital-acquired infection), patient demographics, clinical symptoms and signs, details of hospital course, comorbidities, prior intravenous antibiotic use within 90 days, bacterial cultures, antibiotics sensitivity tests, and clinical outcomes.

\section{Antibiotic susceptibility}

Antibiotic susceptibility was tested using the VITEK ${ }^{\bullet}$ II system with VITEK $^{\circ}$ II Gram Negative Susceptibility cards (bioMérieux, Marcy-l'Étoile, France) with Clinical \& Laboratory Standards Institute interpretive criteria M100-25. One Gram-negative (GN) identification card and another VITEK II AST-N322 card (for susceptibility testing of aerobic GN bacilli against specified antimicrobials) were placed in the neighboring slots, along with the transfer tube and the corresponding suspension tube [29]. The complete list of antibiotics used in susceptibility testing for $A$. faecalis, including piperacillin, piperacillin-tazobactam, ceftazidime, cefepime, cefotaxime, ceftriaxone, ampicillin-sulbactam, imipenem, meropenem, gentamicin, amikacin, ciprofloxacin, levofloxacin in our microbiology laboratory which was reference from clinical and laboratory standards institute (30th edition). Our microbiology laboratory susceptibility test report for A. faecalis included piperacillin-tazobactam, ceftazidime, cefepime, imipenem, meropenem, gentamicin, amikacin, ciprofloxacin, and ampicillin-sulbactam. If the susceptibility test showed resistant to all antibiotics, tigecycline susceptibility in A. faecalis would be done by the disc diffusion method.

We also searched PubMed and google scholar for topics related to Alcaligenes faecalis with no language restrictions.

The definition of MDR/XDR cites the literature of Maggiorakos et al. [30]

\section{Study ethics and consent procedure}

Our study was a retrospective analysis of medical records. The six-year study represented the lowest risk to the research subjects, and all information was made anonymous before being made available for research. The project was approved by the Buddhist Dalin Tzu Chi General Hospital Research Ethics Committee (Approved IRB No. B10802024), which exempted the study from the requirement for informed consent.

\section{Statistical analysis}

Continuous variables were expressed as mean \pm standard deviation or median (range), whereas categorical variables were expressed as frequencies and percentages. The trend in change of antimicrobial susceptibility analyzed by linear-by-linear association in chi-square test. All statistical analyses were conducted using the statistical package SPSS for Windows (Version 17.0, SPSS, Inc., Chicago, IL, USA). A $p$-value $<0.05$ was considered statistically significant.

\section{Results}

Sixty-one cases of $A$. faecalis infection were identified during the study, including 25 cases of cystitis, nine cases of diabetic foot ulcer with infection, eight cases of pneumonia, seven cases of acute pyelonephritis $(\mathrm{APN})$, three cases of bacteremia, and nine cases of infection at specific sites. There were nine hospitalacquired infections cases, including three cases of diabetic foot ulcer with infection, two cases of pneumonia, one case of pleural empyema, one case of peritonitis, one case of surgical wound infection, and one case of leg burn wound infection. Thirty-one community-onset infection cases $(31 / 52=59.6 \%)$ had a history of previous hospitalisation within 90 days. Thirty-seven patients $(60.7 \%)$ had a history of intravenous antibiotic use within the past 90 days. The most commonly used antibiotics were ceftazidime (11 cases) and levofloxacin (ten cases). There were only two cases of receipt of antibiotics during the same hospitalisation but before the onset of $A$. faecalis infection, including one case of prolonged mechanical ventilation with pneumonia and one case of peritonitis. The length of stay before $A$. faecalis isolation of the two cases were 190 days and 22 days, respectively. Fifty-one (83.6\%) cases were mixed with other bacterial infections. The most common mixed infection pathogens were Enterococcus species (nine cases), Proteus vulgaris (nine cases), and Pseudomonas aeruginosa (eight cases).

\section{Cystitis with $A$. faecalis (Table 1)}

Twenty-five cases of $A$. faecalis cystitis were seen during the study period, which accounts for $41 \%$ of A. faecalis infections in this report. There were ten females and 15 males in our study. The mean age was 76.9 years (range: 25 to 98 years). Sixteen cases (64\%) were catheter related cystitis. One patient had 
Table 1 Bacteriology and clinical outcome in twenty-five cases of Alcaligenes faecalis infection cystitis

\begin{tabular}{|c|c|c|c|c|c|c|c|c|c|}
\hline & Case 1 & Case2 & Case 3 & Case 4 & Case 5 & Case 6 & Case7 & Case8 & Case 9 \\
\hline year & 2014 & 2014 & 2014 & 2014 & 2014 & 2014 & 2015 & 2015 & 2015 \\
\hline catheter related cystitis & No & No & Yes & Yes & Yes & Yes & Yes & Yes & Yes \\
\hline $\begin{array}{l}\text { prior intravenous antibiotic use within } 90 \\
\text { days }\end{array}$ & No & No & No & No & FEP & $\begin{array}{l}\text { unknown } \\
\text { (other hospital) }\end{array}$ & No & No & No \\
\hline Antibiotic therapy & MXF & LVX & $\begin{array}{l}\text { LVX } \\
\text { SAM }\end{array}$ & $\begin{array}{l}\text { FEP } \\
\text { LVX }\end{array}$ & ETP & CTX & FEP & MXF & $C A Z$ \\
\hline outcome & cure & cure & cure & cure & cure & dead & dead & cure & cure \\
\hline \multicolumn{10}{|c|}{ Results of antibiotics sensitivity test of Alcaligenes faecalis } \\
\hline GEN & S & S & S & S & S & S & S & S & $\mathrm{R}$ \\
\hline AMK & S & S & S & S & S & S & S & S & $\mathrm{R}$ \\
\hline CAZ & S & S & S & S & S & S & S & S & $\mathrm{S}$ \\
\hline FEP & S & S & S & S & $\mathrm{R}$ & $\mathrm{R}$ & S & S & $\mathrm{R}$ \\
\hline SAM & $S$ & $S$ & $S$ & $S$ & $S$ & $S$ & $S$ & $S$ & $\mathrm{R}$ \\
\hline TZP & $S$ & $S$ & $S$ & $S$ & $S$ & $S$ & $S$ & $S$ & $S$ \\
\hline CIP & $S$ & $S$ & R & $S$ & R & $\mathrm{R}$ & $S$ & $S$ & $\mathrm{R}$ \\
\hline IPM & $S$ & $S$ & $S$ & $S$ & $S$ & $S$ & $S$ & $S$ & S \\
\hline MEM & $S$ & $S$ & $S$ & $S$ & $S$ & $S$ & $S$ & $S$ & $S$ \\
\hline TGC & NA & NA & NA & NA & NA & NA & NA & NA & NA \\
\hline
\end{tabular}

Mixed infection pathogens

Enterococcus spp

Proteus vulgaris

Citrobacter koseri$$
\text { V }
$$

Klebsiella pneumoniae

Providencia rettgeri

Non-fermentative GNB

\begin{tabular}{|c|c|c|c|c|c|c|c|c|}
\hline & $\begin{array}{l}\text { Case } \\
10\end{array}$ & Case 11 & Case 12 & $\begin{array}{l}\text { Case } \\
13\end{array}$ & $\begin{array}{l}\text { Case } \\
14\end{array}$ & Case 15 & $\begin{array}{l}\text { Case } \\
16\end{array}$ & Case 17 \\
\hline year & 2015 & 2015 & 2015 & 2016 & 2016 & 2016 & 2016 & 2016 \\
\hline catheter related cystitis & Yes & No & No & No & Yes & No & Yes & No \\
\hline $\begin{array}{l}\text { prior intravenous antibiotic use within } 90 \\
\text { days }\end{array}$ & No & LVX & $\begin{array}{l}\text { CAZ } \\
\text { CRO }\end{array}$ & LVX & LVX & NO & No & No \\
\hline Antibiotics therapy & $\mathrm{CMZ}$ & SAM & LVX & ETP & $C A Z$ & LVX & LVX & ETP \\
\hline outcome & cure & cure & cure & cure & cure & cure & cure & cure \\
\hline
\end{tabular}

Results of antibiotics sensitivity test of Alcaligenes faecalis

\begin{tabular}{|c|c|c|c|c|c|c|c|c|}
\hline GEN & $S$ & $S$ & S & $\mathrm{R}$ & S & $\mathrm{NIL}$ & $\mathrm{NIL}$ & S \\
\hline AMK & $S$ & $S$ & $S$ & $S$ & $S$ & NIL & $\mathrm{NIL}$ & $S$ \\
\hline CAZ & S & $S$ & S & $S$ & S & $\mathrm{NIL}$ & $\mathrm{NIL}$ & $S$ \\
\hline FEP & S & $S$ & S & S & $S$ & NIL & $\mathrm{NIL}$ & $S$ \\
\hline SAM & S & $S$ & $S$ & $S$ & $S$ & NIL & $\mathrm{NIL}$ & $S$ \\
\hline TZP & $S$ & R & $S$ & $\mathrm{R}$ & $S$ & NIL & $\mathrm{NIL}$ & $\mathrm{R}$ \\
\hline CIP & S & $R$ & $S$ & $\mathrm{R}$ & $\mathrm{R}$ & NIL & $\mathrm{NIL}$ & $\mathrm{R}$ \\
\hline IPM & $S$ & $S$ & $S$ & $S$ & $S$ & $\mathrm{NIL}$ & $\mathrm{NIL}$ & $S$ \\
\hline MEM & $S$ & S & S & S & S & $\mathrm{NIL}$ & $\mathrm{NIL}$ & S \\
\hline TGC & NA & NA & NA & NA & NA & NA & NA & NA \\
\hline
\end{tabular}


Table 1 Bacteriology and clinical outcome in twenty-five cases of Alcaligenes faecalis infection cystitis (Continued)

\begin{tabular}{|c|c|c|c|c|c|c|c|c|c|}
\hline & Case 1 & Case2 & Case 3 & Case 4 & Case 5 & Case 6 & Case7 & Case8 & Case 9 \\
\hline \multicolumn{10}{|l|}{ Mixed infection pathogens } \\
\hline Chryseobacterium meningosepticum & & V & & & & & & & \\
\hline Serratia marcescens & & & & & & V & & & \\
\hline Providencia stuartii & & & & & V & & & V & \\
\hline \multirow[t]{2}{*}{ Escherichia coli } & V & & & V & & & & & \\
\hline & Case18 & Case 19 & Case20 & Case21 & $\begin{array}{l}\text { Case } \\
22\end{array}$ & Case 23 & $\begin{array}{l}\text { Case } \\
24\end{array}$ & Case 25 & \\
\hline year & 2016 & 2017 & 2017 & 2017 & 2018 & 2018 & 2018 & 2019 & \\
\hline catheter related cystitis & Yes & No & Yes & No & Yes & Yes & Yes & Yes & \\
\hline $\begin{array}{l}\text { prior intravenous antibiotic use within } 90 \\
\text { days }\end{array}$ & $\begin{array}{l}\text { CRO } \\
\text { CFZ }\end{array}$ & $\begin{array}{l}\text { Unknow } \\
\text { (other Hospital) }\end{array}$ & LVX & $\begin{array}{l}\text { CFZ } \\
\text { CMZ }\end{array}$ & $\begin{array}{l}\text { LVX } \\
\text { FEP }\end{array}$ & $\begin{array}{l}\text { Unknow } \\
\text { (other Hospital) }\end{array}$ & $\begin{array}{l}\text { FEP } \\
\text { TEC }\end{array}$ & $\begin{array}{l}\text { Unknow } \\
\text { (other Hospital) }\end{array}$ & \\
\hline Antibiotics therapy & SAM & LVX & CAZ & CAZ & LVX & MEM & FEP & FEP & \\
\hline outcome & cure & cure & cure & cure & cure & cure & cure & cure & \\
\hline \multicolumn{10}{|c|}{ Results of antibiotics sensitivity test of Alcaligenes faecalis } \\
\hline GEN & S & $S$ & S & S & S & S & S & $\mathrm{S}$ & \\
\hline AMK & S & S & S & S & S & S & S & S & \\
\hline CAZ & S & S & S & S & S & S & S & S & \\
\hline FEP & S & S & S & S & S & $\mathrm{S}$ & S & S & \\
\hline SAM & S & S & S & S & S & S & S & S & \\
\hline TZP & R & S & R & R & S & $\mathrm{R}$ & R & $\mathrm{R}$ & \\
\hline CIP & $\mathrm{R}$ & $S$ & $\mathrm{R}$ & R & S & R & $\mathrm{R}$ & S & \\
\hline IPM & S & $S$ & S & S & S & $\mathrm{S}$ & S & $\mathrm{S}$ & \\
\hline MEM & S & $\mathrm{S}$ & S & S & S & S & S & S & \\
\hline TGC & NA & NA & NA & NA & NA & NA & NA & NA & \\
\hline \multicolumn{10}{|l|}{ Mixed infection pathogens } \\
\hline Proteus vulgaris & & & & & & & & V & \\
\hline Enterobacter cloacae & V & & & & & & & & \\
\hline Pseudomonas aeruginosa & & V & & & & & & & \\
\hline Providencia stuartii & & & & & & & V & & \\
\hline Morganella morganii & & & V & & & & & & \\
\hline Enterococcus & & & & & V & & & & \\
\hline Serratia marcescens & & & & V & & & & & \\
\hline
\end{tabular}

no comorbidity, while 24 patients had comorbidities such as diabetes mellitus, hypertension, dementia, cerebrovascular accident and chronic kidney disease (supplement). The most common was neurologic comorbidity (18 cases). Fourteen patients had a history of intravenous antibiotic exposure within the past 90 days. Nineteen patient urine cultures displayed polymicrobial infection. The most common mixed infectious pathogen was Providencia species (five cases). The antibiotics sensitivity test showed no presence of extensively drug-resistant (XDR) A. faecalis. Two patients died from urosepsis. Twentythree patients were treated with adequate antibiotics therapy and were discharged in excellent condition.

\section{Acute pyelonephritis (Table 2)}

Seven patients (six females and one male) with A. faecalis acute pyelonephritis were seen during the study. The mean age was 75.4 years (range: 63 to 85 years). All seven patients had a risk of obstructive uropathy (four cases of cervical carcinoma, two cases of renal stones, and one case of right ureteral cancer). Four patients had a history of intravenous antibiotic exposure within the past 90 days. Six patient urine cultures displayed polymicrobial infection. The most common mixed infectious pathogen was Providencia rettgeri, which affected three patients. The antibiotic sensitivity test showed no presence of XDR A. faecalis. All patients received adequate IV antibiotic therapy, and all were discharged from the hospital in excellent condition. 
Table 2 Bacteriology and clinical outcome in seven cases of Alcaligenes faecalis acute pyelonephritis

\begin{tabular}{|c|c|c|c|c|c|c|c|}
\hline & Case 1 & Case 2 & Case 3 & Case 4 & Case 5 & Case 6 & Case7 \\
\hline year & 2014 & 2014 & 2015 & 2015 & 2016 & 2017 & 2017 \\
\hline prior intravenous antibiotic use within 90 days & No & Unknown (other hospital) & no & $\begin{array}{l}\text { ETP } \\
\text { LVX } \\
\text { CMZ }\end{array}$ & No & $\begin{array}{l}\text { ETP } \\
\text { TEC } \\
\text { CMZ }\end{array}$ & $\begin{array}{l}\text { AMC } \\
\text { ETP } \\
\text { CFZ }\end{array}$ \\
\hline Antibiotics therapy & SAM & CIP & $\mathrm{CIP}$ & LVX & ETP & ETP & $\begin{array}{l}\text { TEC } \\
\text { ERT }\end{array}$ \\
\hline outcome & cure & cure & cure & cure & cure & cure & cure \\
\hline \multicolumn{8}{|c|}{ Results of antibiotics sensitivity test of Alcaligenes faecalis } \\
\hline GEN & S & S & S & $S$ & S & $S$ & S \\
\hline AMK & S & S & S & S & S & S & S \\
\hline CAZ & S & S & S & S & S & S & S \\
\hline FEP & $S$ & S & S & S & S & S & S \\
\hline SAM & S & S & S & S & S & S & S \\
\hline TZP & S & S & S & $S$ & S & $\mathrm{R}$ & S \\
\hline $\mathrm{CIP}$ & $\mathrm{R}$ & S & S & $S$ & $\mathrm{R}$ & $\mathrm{R}$ & $\mathrm{R}$ \\
\hline IPM & S & S & S & $S$ & S & S & S \\
\hline MEM & S & S & S & S & S & $\mathrm{R}$ & S \\
\hline TGC & NA & NA & NA & NA & NA & NA & NA \\
\hline \multicolumn{8}{|l|}{ Mixed infection pathogens } \\
\hline Proteus vulgaris & & & & & V & & V \\
\hline Escherichia coli & & & & & V & & \\
\hline Klebsiella pneumoniae & & & & & & V & \\
\hline Providencia rettgeri & & V & V & & & V & \\
\hline enterococcus & & & & V & & & \\
\hline
\end{tabular}

\section{Diabetic foot ulcer with $A$. faecalis infection (Table 3 )}

Six male patients and three female patients had diabetic foot ulcers with $A$. faecalis infection. The mean age was 57.2 years (range: 41 to 85 years). All patients had other comorbidities. All patients' foot ulcer lesions were chronic (range: 14 days to 18 months). Two patients had no history of prior intravenous antibiotic use within 90 days. All patients' wound cultures displayed polymicrobial infection. The antibiotics sensitivity test showed the presence of XDR A. faecalis infection beginning in 2019. All patients required surgical intervention. The wounds did not heal in three patients.

\section{Pneumonia (Table 4)}

Eight cases (six males, two females) of $A$. faecalis pneumonia were seen during the study period. The mean age was 70.0 years (range: $51-83$ years). All eight patients were at risk of pneumonia (three patients had malignancies, one had end-stage renal disease, and four patients were bed-ridden with neurologic deficits). One patient had no history of prior intravenous antibiotic use within 90 days. Six patient sputum cultures displayed polymicrobial infection. The antibiotics sensitivity test showed the presence of XDR A. faecalis beginning in 2018. Two patients died from $A$. faecalis pneumonia. Six patients received adequate intravenous antibiotics therapy and were discharged in excellent condition.

\section{Bacteremia (no concurrent primary site of infection)}

Three patients developed bacteremia during the study period. These three patients included a 78-year-old male, a bedridden stroke survivor; an 81-year-old male, a bedridden dementia; and an 81-year-old female with cholangiocarcinoma and dementia. The two male patients had a history of intravenous antibiotic exposure within the past 90 days. Two patients' blood cultures displayed polymicrobial infection (one patient had a mixed infection with Enterococcus faecalis, and another patient had a mixed infection with Morganella morganii). Antibiotic sensitivity tests showed no presence of XDR A. faecalis. All patients received adequate antibiotics therapy and were discharged in excellent condition. Nine specific sites of $A$. faecalis infection cases are shown in Table 5. The trend in change of antimicrobial susceptibility analyzed by linear-by-linear association in chi-square test (Table 6). The susceptibility rate of $A$. faecalis to commonly used antibiotics (except ciprofloxacin and cefepime) is decreasing year by year. 
Table 3 Bacteriology and clinical outcome in nine cases of diabetic foot ulcer with Alcaligenes faecalis infection

\begin{tabular}{|c|c|c|c|c|c|c|c|c|c|}
\hline & Case 1 & Case2 & Case 3 & Case 4 & Case 5 & Case 6 & Case7 & Case8 & Case9 \\
\hline year & 2014 & 2015 & 2015 & 2017 & 2018 & 2018 & 2019 & 2019 & 2019 \\
\hline prior intravenous antibiotic use within 90 days & $\begin{array}{l}\text { CIP } \\
\text { SAM }\end{array}$ & $\begin{array}{l}\text { PIP } \\
\text { CRO } \\
\text { VAN }\end{array}$ & No & $\begin{array}{l}\text { CFZ } \\
\text { TEC }\end{array}$ & No & $\begin{array}{l}\text { CAZ } \\
\text { CIP } \\
\text { PIP } \\
\text { TEC }\end{array}$ & $\begin{array}{l}\text { CAZ } \\
\text { VAN }\end{array}$ & $\begin{array}{l}\text { LVX } \\
\text { MEM } \\
\text { TEC }\end{array}$ & $\begin{array}{l}\text { CRO } \\
\text { CAZ } \\
\text { OXA }\end{array}$ \\
\hline Antibiotic therapy & SAM & $\begin{array}{l}\text { MEM } \\
\text { VAN }\end{array}$ & $\begin{array}{l}\text { VAN } \\
\text { CAZ }\end{array}$ & $\begin{array}{l}\text { OXA } \\
\text { CMZ }\end{array}$ & $\begin{array}{l}\text { MEM } \\
\text { VAN }\end{array}$ & $\begin{array}{l}\text { CAZ } \\
\text { TEC }\end{array}$ & $\begin{array}{l}\text { CAZ } \\
\text { VAN }\end{array}$ & CST & $\begin{array}{l}\text { CAZ } \\
\text { MEM }\end{array}$ \\
\hline outcome & heal & heal & heal & heal & no heal & no heal & heal & no heal & heal \\
\hline \multicolumn{10}{|c|}{ Results of antibiotics sensitivity test of Alcaligenes faecalis } \\
\hline GEN & S & S & S & $\mathrm{R}$ & $S$ & 1 & $\mathrm{R}$ & $\mathrm{R}$ & $S$ \\
\hline AMK & $S$ & S & $S$ & S & $S$ & $S$ & $R$ & $\mathrm{R}$ & $S$ \\
\hline CAZ & $S$ & $S$ & $S$ & $S$ & $S$ & $S$ & $\mathrm{R}$ & $R$ & $S$ \\
\hline FEP & $S$ & $S$ & $S$ & I & $S$ & 1 & $\mathrm{R}$ & $\mathrm{R}$ & $S$ \\
\hline SAM & $S$ & $S$ & $S$ & 1 & $S$ & $S$ & $\mathrm{R}$ & $\mathrm{R}$ & $\mathrm{S}$ \\
\hline TZP & $S$ & $S$ & $S$ & 1 & S & $S$ & $R$ & $\mathrm{R}$ & $\mathrm{R}$ \\
\hline CIP & S & S & $S$ & $\mathrm{R}$ & $S$ & $\mathrm{R}$ & $\mathrm{R}$ & R & $S$ \\
\hline IPM & $S$ & $S$ & $S$ & $S$ & $S$ & $S$ & $\mathrm{R}$ & $\mathrm{R}$ & $S$ \\
\hline MEM & $S$ & $S$ & $S$ & $S$ & $S$ & $S$ & R & $\mathrm{R}$ & $S$ \\
\hline TGC & NA & NA & NA & NA & NA & NA & $S$ & $S$ & NA \\
\hline \multicolumn{10}{|l|}{ Mixed infection pathogens } \\
\hline Enterococcus spp & V & & & & V & & & V & V \\
\hline Proteus vulgaris & & V & & & V & & & & \\
\hline Citrobacter koseri & & & V & & & & & & \\
\hline MRSA & & & V & & & & & & \\
\hline Klebsiella pneumoniae & & & & & & V & V & V & \\
\hline Pseudomonas aeruginosa & & & & & & & & & V \\
\hline Providencia rettgeri & & & & & & V & & & \\
\hline Acinetobacter baumannii & & & & & & & V & V & \\
\hline Morganella morganii & & & & V & & & & & \\
\hline
\end{tabular}

\section{Discussion and literature review}

According to the literature, there were 130 sporadically reported cases of $A$. faecalis infection (Table 7) [1, 2, 7$28,31-46]$. The most commonly reported cases involved bacteremia, and most cases occurred in newborns and infants. In 1960, Doxiadis reported 33 cases of bacteremia in newborns, which was the largest case series of $A$. faecalis bacteremia [40]. A. faecalis was resistant to sulfonamides, and there were 20 deaths due to A. faecalis bacteremia. Fillipe reported 20 cases of chronic otitis media in Angola [19]. The use of bird feces by residents as a traditional remedy to prevent ear discharge was related to these $A$. faecalis chronic otitis media cases. The other infections from A. faecalis that have been reported in prior studies, in order of occurrence, were meningitis, skin and soft tissue infection (SSTI), and UTI. In our series, the most frequent cases were, in order of occurrence, UTI, SSTI, and pneumonia. The cases reported in the literature and our cases indicate that the most frequent $A$. faecalis infection sites, in order, are the bloodstream, urinary tract, skin and soft tissue (diabetic foot ulcer accounts for $56.5 \%$ of skin and soft tissue infections), and middle ear.

\section{A. faecalis isolation in mixed culture as a pathogen or contaminant}

In Tena's report, two out of five skin and soft tissue $A$. faecalis cases were mixed with other bacterial infections [17]. In Filipe's series, all 20 A. faecalis otitis media cases were mixed with other bacterial infections [19]. Kahveci reported a case of $A$. faecalis peritonitis and concluded that it was important to view A. faecalis as a pathogen rather than a contaminant [15]. Junejo notes that it is evident that any organism found in the culture should not be completely disregarded and marked as a contaminant [23].. Al-Zakhari explains that serious illnesses and 
Table 4 Bacteriology and clinical outcome in eight cases of Alcaligenes faecalis pneumonia

\begin{tabular}{|c|c|c|c|c|c|c|c|c|}
\hline & Case 1 & Case 2 & Case 3 & Case 4 & Case 5 & Case 6 & Case7 & Case8 \\
\hline year & 2014 & 2014 & 2015 & 2018 & 2019 & 2019 & 2019 & 2019 \\
\hline prior intravenous antibiotic use within 90 days & No & $\begin{array}{l}\text { ETP } \\
\text { FEP } \\
\text { VAN }\end{array}$ & LVX & $\begin{array}{l}\text { CAZ } \\
\text { LVX } \\
\text { DOR } \\
\text { VAN }\end{array}$ & $\begin{array}{l}\text { CAZ } \\
\text { AMK } \\
\text { IPM } \\
\text { TEC } \\
\text { CIP }\end{array}$ & $\begin{array}{l}\text { TZP } \\
\text { MEM } \\
\text { CAZ } \\
\text { TEC } \\
\text { AMK }\end{array}$ & $\begin{array}{l}\text { CRO } \\
\text { SAM }\end{array}$ & $\begin{array}{l}\text { TZP } \\
\text { MEM } \\
\text { CAZ } \\
\text { VAN } \\
\text { LVX } \\
\text { AMK }\end{array}$ \\
\hline Antibiotics therapy & CXM & CRO & FEP & TGC & TGC & MEM & CAZ & CAZ \\
\hline outcome & cure & cure & dead & cure & cure & cure & dead & cure \\
\hline \multicolumn{9}{|c|}{ Results of antibiotics sensitivity test of Alcaligenes faecalis } \\
\hline GEN & S & S & $\mathrm{R}$ & $\mathrm{R}$ & $\mathrm{R}$ & $\mathrm{R}$ & S & NIL \\
\hline AMK & $S$ & S & $\mathrm{R}$ & R & $\mathrm{R}$ & $\mathrm{R}$ & S & $\mathrm{NIL}$ \\
\hline CAZ & S & S & $\mathrm{R}$ & $\mathrm{R}$ & $\mathrm{R}$ & S & S & $\mathrm{NIL}$ \\
\hline FEP & S & $S$ & $\mathrm{R}$ & $\mathrm{R}$ & $\mathrm{R}$ & $\mathrm{R}$ & S & $\mathrm{NIL}$ \\
\hline SAM & S & S & $\mathrm{R}$ & $\mathrm{R}$ & $\mathrm{R}$ & $\mathrm{R}$ & S & $\mathrm{NIL}$ \\
\hline TZP & S & S & $\mathrm{R}$ & $\mathrm{R}$ & $\mathrm{R}$ & S & $\mathrm{R}$ & $\mathrm{NIL}$ \\
\hline CIP & S & S & $\mathrm{R}$ & $\mathrm{R}$ & $\mathrm{R}$ & $\mathrm{R}$ & $\mathrm{R}$ & $\mathrm{NIL}$ \\
\hline IPM & S & S & S & $\mathrm{R}$ & R & S & S & $\mathrm{NIL}$ \\
\hline MEM & S & S & S & $\mathrm{R}$ & $\mathrm{R}$ & S & S & $\mathrm{NIL}$ \\
\hline TGC & NA & NA & NA & S & S & NA & NA & NA \\
\hline \multicolumn{9}{|l|}{ Mixed infection pathogens } \\
\hline Proteus vulgaris & V & & & & & & & \\
\hline MSSA & & & & & & & V & \\
\hline Pseudomonas aeruginosa & & & & & & V & & V \\
\hline Providencia stuartii & & V & & & & & & \\
\hline Brukholderia cepacia & & & V & & & & & \\
\hline
\end{tabular}

even death can be caused by $A$. faecalis. Therefore, rather than a contaminant, A. faecalis should be regarded as a pathogen, because global cases of life-threatening infections caused by $A$. faecalis are emerging [27]. In 2017, Laham reported a clinical sample study of an $A$. faecalis strain isolated from two outpatients and three inpatients, including four wounds cultures and one urine culture [47]. However, this study was only conducted for a three-month period. In 2013, Khajuria reported a total of 15 clinical isolates of $A$. faecalis specimens such as urine, pus, blood, and body fluids [48]. We believe that many cases of $A$. faecalis infections cases exist but have not been reported in the literature. Our series of $A$. faecalis infection cases were about 10 cases every year, which was only a small fraction of the infectious diseases in our hospital. We concur with Junejo and believe $A$. faecalis to be an infectious pathogen rather than contaminant. In our series, the majority of cultures were mixed with other well-established pathogens. However, one should consider that $A$. faecalis may be a contaminant in some cases, particularly for those who were cured despite lack of active treatment.
The trend of antibiotic sensitive rate of $A$. faecalis

In 1997, Bizet first reported that $A$. faecalis strains were resistant to amoxicillin, ticarcillin, and gentamicin [1]. Amoxicillin-clavulanic acid and cefotaxime provided a successful treatment outcome for patients with A. faecalis infection. In 2000, Pereira reported that a strain of $A$. faecalis resistant to expanded-spectrum beta-lactamase cephalosporins was isolated from the urine of an inpatient [2]. In 2005, Dubois described the isolation of $A$. faecalis with ESBL in a patient with a concurrent urinary tract infection [11]. In 2017 and 2018, two cases of XDR A. faecalis pneumonia were reported by Agarwal and Junejo [22, 23]. In 2019, Hasan reported that a 60-yearold female with pandrug-resistant $A$. faecalis bacteremia who was treated with double-dose tigecycline had a successful treatment outcome [26]. Three articles on pandrug-resistant $A$. faecalis were published in 2020. AlZakhari reported a fatal case of cavitary pneumonia caused by pandrug-resistant $A$. faecalis. The patient died despite the aggressive antibiotic treatment (linezolid and polymyxin B) [27]. Majewski reported a pandrugresistant A. faecalis hospital acquired urinary tract 
Table 5 Bacteriology and clinical outcome in nine cases with Alcaligenes faecalis infection

\begin{tabular}{|c|c|c|c|c|c|c|c|c|c|}
\hline & Case 1 & Case2 & Case 3 & Case 4 & Case 5 & Case 6 & Case7 & Case8 & Case9 \\
\hline year & 2016 & 2018 & 2017 & 2019 & 2015 & 2016 & 2016 & 2016 & 2019 \\
\hline prior intravenous antibiotic use within 90 days & $\mathrm{NIL}$ & AMC & No & No & $\begin{array}{l}\text { CAZ } \\
\text { CIP } \\
\text { MEM }\end{array}$ & No & No & CFZ & No \\
\hline Antibiotics therapy & $\begin{array}{l}\text { MXF } \\
\text { AMC }\end{array}$ & CAZ & OFX & SXT & CAZ & CFZ & СТВ & CFZ & $\begin{array}{l}\text { TEC } \\
\text { CRO }\end{array}$ \\
\hline outcome & cure & cure & cure & cure & cure & cure & cure & cure & cure \\
\hline \multicolumn{10}{|c|}{ Results of antibiotics sensitivity test of Alcaligenes faecalis } \\
\hline GEN & $\mathrm{R}$ & S & $S$ & S & S & $\mathrm{R}$ & NIL & NIL & S \\
\hline AMK & S & $\mathrm{S}$ & S & S & S & S & $\mathrm{NIL}$ & $\mathrm{NIL}$ & S \\
\hline CAZ & $\mathrm{R}$ & S & S & S & S & $\mathrm{R}$ & $\mathrm{NIL}$ & $\mathrm{NIL}$ & S \\
\hline FEP & $\mathrm{R}$ & S & $S$ & S & S & $\mathrm{R}$ & NIL & NIL & S \\
\hline SAM & $\mathrm{R}$ & $S$ & $S$ & S & S & $\mathrm{R}$ & NIL & NIL & S \\
\hline TZP & $\mathrm{R}$ & S & $S$ & S & S & $\mathrm{R}$ & NIL & NIL & S \\
\hline $\mathrm{CIP}$ & $\mathrm{R}$ & S & S & $\mathrm{R}$ & $\mathrm{R}$ & S & $\mathrm{NIL}$ & NIL & S \\
\hline IPM & S & S & S & S & S & S & NIL & NIL & S \\
\hline MEM & S & $\mathrm{S}$ & S & S & S & S & NIL & $\mathrm{NIL}$ & S \\
\hline TGC & NA & NA & NA & NA & NA & NA & NA & NA & NA \\
\hline \multicolumn{10}{|l|}{ Mixed infection pathogens } \\
\hline Enterococcus & & & & & & & & & V \\
\hline Proteus vulgaris & & & & & & & & V & V \\
\hline Enterobacter cloacae & & V & & & V & & & & \\
\hline Pseudomonas aeruginosa & V & & V & & & & & V & V \\
\hline Acinetobacter baumannii & & V & & & & & & & \\
\hline Escherichia coli & & & & & & & V & & \\
\hline Serratia marcescens & & & & V & & & & & \\
\hline Providencia stuartii & V & & & & & & & & \\
\hline MSSA & & & & V & & & & & \\
\hline Streptococcus group D & & & & & & & V & & \\
\hline Aeromonas hydrophila & & & & & & V & & & \\
\hline
\end{tabular}

Case 1 and case 2: Pleural empyema, Case 3 and case 4: otitis media, Case 5: peritonitis, Case 6: surgical wound infection,

Case 7: pelvis abscess, Case 8: scrotum abscess, Case 9: burn wound infection

Table 6 The trend in change of antimicrobial susceptibility to Alcaligenes faecalis analyzed by linear-by-linear association in chisquare test

\begin{tabular}{lllllllll}
\hline Antibiotics/year (number of patients) & $\mathbf{2 0 1 4}(\mathbf{1 2})$ & $\mathbf{2 0 1 5}(\mathbf{1 3 )}$ & $\mathbf{2 0 1 6} \mathbf{( 9 )}$ & $\mathbf{2 0 1 7}(\mathbf{9 )}$ & $\begin{array}{l}\mathbf{2 0 1 8} \\
\mathbf{( 7 )}\end{array}$ & $\mathbf{2 0 1 9}(\mathbf{9 )}$ & $\mathbf{M}^{\mathbf{2}}$ & $\boldsymbol{P}_{\text {value }}$ \\
\hline CAZ & $100 \%$ & $92.3 \%$ & $77.8 \%$ & $88.7 \%$ & $85.7 \%$ & $66.7 \%$ & 4.063 & 0.044 \\
GEN & $100 \%$ & $84.6 \%$ & $55.6 \%$ & $77.8 \%$ & $71.4 \%$ & $55.6 \%$ & 5.144 & 0.023 \\
AMK & $100 \%$ & $84.6 \%$ & $100 \%$ & $100 \%$ & $85.7 \%$ & $55.6 \%$ & 5.837 & 0.016 \\
CIP & $50.0 \%$ & $61.5 \%$ & $22.2 \%$ & $22.2 \%$ & $42.9 \%$ & $33.3 \%$ & 1.589 & 0.207 \\
IMP & $100 \%$ & $100 \%$ & $100 \%$ & $100 \%$ & $85.7 \%$ & $66.7 \%$ & 9.042 & 0.003 \\
SAM & $100 \%$ & $84.6 \%$ & $77.8 \%$ & $88.7 \%$ & $85.7 \%$ & $55.6 \%$ & 4.622 & 0.032 \\
FEP & $83.3 \%$ & $76.9 \%$ & $77.8 \%$ & $66.7 \%$ & $71.4 \%$ & $55.6 \%$ & 2.028 & 0.154 \\
MEM & $100 \%$ & $100 \%$ & $100 \%$ & $100 \%$ & $85.7 \%$ & $66.7 \%$ & 9.042 & 0.003 \\
TZP & $100 \%$ & $84.6 \%$ & $33.3 \%$ & $42.4 \%$ & $57.1 \%$ & $33.3 \%$ & 11.713 & 0.001 \\
\hline
\end{tabular}


Table 7 Alcaligenes faecalis infection cases

\begin{tabular}{llll}
\hline Year/diagnosis & Cases of literature & Our cases & total \\
\hline Cystitis & 11 & 25 & 36 \\
Skin and soft tissue infection & 12 & $11^{*}$ & 23 \\
Pneumonia & 7 & 8 & 15 \\
Acute pyelonephritis & 0 & 7 & 7 \\
Bacteremia & 42 & 3 & 45 \\
Pleural empyema & 0 & 2 & 2 \\
Otitis media & 21 & 2 & 23 \\
Meningitis & 16 & 0 & 16 \\
Endocarditis & 3 & 0 & 3 \\
Ocular infection & 10 & 0 & 10 \\
Peritonitis & 2 & 1 & 3 \\
Infectious diarrhea & 3 & 0 & 3 \\
specific sites infection & 3 & 2 & 5 \\
Total & 130 & 61 & 191 \\
\hline
\end{tabular}

*: including 9 cases of diabetic foot infection, 1 case of surgical wound infection and 1 case of burn wound infection

infection patient; the patient died in hospital [28]. Ngbede identified mobile colistin resistance genes in Alcaligenes faecalis from human clinical samples [49].

In March 2015, the strain A. faecalis exhibited sensitivity only to imipenem and meropenem in a pneumonia patient in our hospital. In May 2018, a strain of XDR A. faecalis susceptible only to tigecycline was isolated from a pneumonia patient. There were four cases with XDR A. faecalis infection in our series, including two cases of pneumonia and two cases of diabetic foot infection.

In view of individual antibiotics, ciprofloxacin revealed a very low susceptibility rate of $A$. faecalis from 2014 to 2019. Piperacillin/tazobactam was effective in significantly decreasing the susceptibility rate of A. faecalis since 2016. Emerging resistant strains of $A$. faecalis to imipenem and meropenem have been found since 2018. A high resistance rate of many antibiotics was also found in 2019. The best sensitivity rate to $A$. faecalis was $66.7 \%$ for three antibiotics (imipenem, meropenem, and ceftazidime). In two antibiotics (ciprofloxacin and piperacillin/tazobactam) sensitivity rates to A. faecalis were less than $50 \%$.

Based on our prior experience, we selected an appropriate antibiotic for a susceptible $A$. faecalis infection patient according to the results of his or her antibiotics sensitivity test. If the A. faecalis organism is an ESBL strain, carbapenem is an appropriate antibiotic. Four articles mentioned that $A$. faecalis is susceptible to colistin $[8,19,22,23]$. Data was not available regarding $A$. faecalis susceptibility to colistin in this study. If the A. faecalis is an XDR strain, we recommend that tigecycline is effective to XDR A. faecalis.
Treatment failure of $A$. faecalis infection cases

Among our reported cases of A. faecalis infection, there were seven treatment failure cases, including two cases of pneumonia, two cases of cystitis complicated with sepsis, and three cases of diabetic foot infection. The overall treatment failure rate was $11.5 \%$. A. faecalis is a low virulence bacterium. With adequate intravenous antibiotic therapy, patients with $A$. faecalis infection will typically experience a positive treatment outcome.

\section{Patients were cured with non-covering regimens}

Four cases were cured with non-covering antibiotics, including two cases of diabetic foot infection, one case of pleural empyema, and one case of surgical wound infection. The four cases had received appropriate wound care, adequate abscess drainage, and surgical intervention which may be crucial for curing of infections.

\section{Limitations}

Our clinical study of $A$. faecalis infection was a small case series and therefore can provide only minimal clinical experience. Additional case series reports of $A$. faecalis infection will add to the knowledge of how to treat A. faecalis infection. Misidentification of Acinetobacter baumannii as Alcaligenes faecalis by VITEK II system was reported in the literature [50]. Matrix-assisted laser desorption ionization time of flight mass spectrometry and 16S rRNA sequencing are helpful for the accurate identification between these two species [51]. However, we cannot perform the two methods to confirm the isolates in our hospital. If the culture is XDR A. faecalis, matrix-assisted laser desorption ionization time of flight mass spectrometry and 16S rRNA sequencing can be performed. An accurate distinction between Acinetobacter baumannii and $A$. faecalis has substantial clinical significance.

\section{Conclusions}

A. faecalis exhibited decreasing sensitivity rate to commonly used antibiotics in 2019. Extensively drugresistant Alcaligenes faecalis infections have emerged recently. We select an antibiotic for patients susceptible to A. faecalis infection based on the results of the antibiotics sensitivity test. With adequate intravenous antibiotic therapy, patients with $A$. faecalis infection will typically experience a positive treatment outcome.

\section{Supplementary Information}

The online version contains supplementary material available at https://doi. org/10.1186/s12879-020-05557-8.

Additional file 1. 


\section{Abbreviations}

AMP: Ampicillin; SAM: Ampicillin-sulbactam; AMX: Amoxicillin; AMC: Amoxycillin-clavulanic acid; CFZ: Cefazolin; CMZ: Cefmetazole; CXM: Cefuroxime; CRO: Ceftriaxone; CTX: Cefotaxime; CTB: Ceftibuten; CAZ: Ceftazidime; FEP: Cefepime; GEN: Gentamicin; AMK: Amikacin; OFX: Ofloxacin; CIP: Ciprofloxacin; MXF: Moxifloxacin; LVX: Levofloxacin; ETP: Ertapenem; IMP: Imipenem; MEM: Meropenem; DOR: Doripenem; TZP: Piperacillin-tazobactam; OXA: Oxacillin; VAN: Vancomycin; TEC: Teicoplanin; CST: Colistin; TGC: Tigecycline; SXT: Trimethoprimsulfamethoxazole; MDR: Multidrug-resistant; XDR: Extensively drug-resistant; PDR: Pandrug-resistant; ESBL: Extended-spectrum $\beta$-lactamase; S: Sensitive; R: Resistant; I: Intermediate; VRE: Vancomycin resistant enterococcus; MSSA: Methicillin sensitive Staphylococcus aureus; MRSA: Methicillin resistant Staphylococcus aureus; GNB: Gram-negative bacillus; NA: Not accessed; NIL: No data available

\section{Acknowledgements}

Not applicable.

\section{Author's contributions}

$\mathrm{CH}$ designed the study, collected the data, analyzed the data, wrote the manuscript, and reviewed the manuscript. The author(s) read and approved the final manuscript.

\section{Funding}

There was no funding in this study.

\section{Availability of data and materials}

The raw data available upon reasonable request from the corresponding authors.

\section{Ethics approval and consent to participate}

Our study is a retrospective medical records data collection and analysis. The 6-year study represented the lowest risk to the research subjects, and all information was made anonymous before being made available for research. The project was approved by the Buddhist Dalin Tzu Chi General Hospital Research Ethics Committee (Approved IRB No. B10802024), which exempted the study from the requirement for informed consent.

\section{Consent for publication}

Not applicable.

\section{Competing interests}

The authors declare that they have no competing interests.

\section{Received: 7 May 2020 Accepted: 29 October 2020}

\section{Published online: 11 November 2020}

\section{References}

1. Bizet J, Bizet C. Strains of Alcaligenes faecalis from clinical material. J Inf Secur. 1997;35:167-9.

2. Pereira M, Perilli M, Mantengoli E, Luzzaro F, Toniolo A, Rossolini GM. PER-1 extended-spectrum beta-lactamase production in an Alcaligenes faecalis clinical isolate resistant to expanded-spectrum cephalosporins and monobactams from a hospital in northern Italy. Microb Drug Resist. 2000;6: 85-90. https://doi.org/10.1089/mdr.2000.6.85.

3. Dunne WM, Maisch S. Epidemiological investigation of infections due to Alcaligenes species in children and patients with cystic fibrosis: use of repetitive-element-sequence polymerase chain reaction. Clin Infect Dis. 1995;20:836-41.

4. Mordi RM, Yusuf EO, Onemu SO, Igeleke CL, Odjadjare EE. The prevalence of Alcaligenes faecalis in bacteremia, meningitis and wound sepsis in a tertiary health care institution in western part of Nigeria. Int J Biotechnol. 2013;2: 123-9.

5. Mordi RM, Burke ME, Odjadjare EE, Enabulelen SA, Umeh OJ. Prevalence of urinary tract infections among pregnant women in university of Benin teaching hospital ( UBTH) Benin City, Nigeria. J Asian Sci Res. 2015;5(4):198-204.

6. Siddiqui N, Kirmani S, Khan F, Kaushal N, Shukla IK, Khan HM, et al. Prevalence and Risk Factors of Candida blood Stream Infections in a Tertiary Care Hospital. Int J Curr Microbiol App Sci. 2015;(Special Issue-1):157-62.
7. Aste N, Atzori L, Zucca M, Pau M, Biggio P. Gram-negative bacterial toe web infection: a survey of 123 cases from the district of Cagliari, Italy. J Am Acad Dermatol. 2001;45:537-41. https://doi.org/10.1067/mjd.2001.114747.

8. Khokhar DS, Sethi HS, Kumar H, Sudan R, Sharma N, Niranjan N. Postkeratoplasty endophthalmitis by Alcaligenes faecalis: a case report. Cornea. 2002;21:232-3.

9. Aisenberg G, Rolston KV, Safdar A. Bacteremia caused by Achromobacter and Alcaligenes species in 46 patients with cancer (1989-2003). Cancer. 2004 Nov 1;101(9):2134-40.

10. Ashwath ML, Katner HP. Pancreatic abscess secondary to Alcaligenes faecalis. Am J Med Sci. 2005;329:54-5.

11. Dubois V, Arpin C, Coulange L, André C, Noury P, Quentin C, et al. TEM-21 extended-spectrum beta-lactamase in a clinical isolate of Alcaligenes faecalis from a nursing home. J Antimicrob Chemother. 2006;57:368-9. https://doi.org/10.1093/jac/dki450.

12. Leesik H, Ani U, Juhani A, Altraja A. Microbial pathogens of adult communityacquired pneumonia in Southern Estonia. Medicina (Kaunas). 2006;42(5):384-94.

13. Kaliaperumal S, Srinivasan R, Gupta A, Parija SC. Postoperative endophthalmitis due to an unusual pathogen: Alcaligenes faecalis. Eye (Lond). 2006;20:968-9. https://doi.org/10.1038/sj.eye.6702080.

14. Kavuncuoglu F, Unal A, Oguzhan N, Tokgoz B, Oymak O, Utas C. First reported case of Alcaligenes faecalis peritonitis. J Int Soc Perit Dial. 2010;1: 118-9. https://doi.org/10.3747/pdi.2009.00058.

15. Kahveci A, Asicioglu E, Tigen E, Ari E, Arikan H, Odabasi Z, et al. Unusual causes of peritonitis in a peritoneal dialysis patient: Alcaligenes faecalis and Pantoea agglomerans. Ann Clin Microbiol Antimicrob. 2011;10:12. https:// doi.org/10.1186/1476-0711-10-2.

16. Pal SS, Panigrahi PK, Roy R, Nandi K, Das S. Endophthalmitis caused by Alcaligenes faecalis: a case series. Ocul Immunol Inflamm. 2013;21:446-8. https://doi.org/10.3109/09273948.

17. Tena D, Fernández C, Lago MR. Alcaligenes faecalis: an unusual cause of skin and soft tissue infection. Jpn J Infect Dis. 2015;68:128-30.

18. Prasad A, Anwer $S$, Qadeer $U$, Martine C. Acute embolic infarcts from Alcaligenes Faecalis meningitis: a case report. J Neuroinfect Dis. 2016;7:1.

19. Filipe M, Reimer Å, Matuschek E, Paul M, Pelkonen T, Riesbeck K. Fluoroquinolone-resistant Alcaligenes faecalis related to chronic suppurative otitis media, Angola. Emerg Infect Dis. 2017;23:1740-2. https://doi.org/10. 3201/eid2310.170268.

20. Chu AS, Harkness J. Alcaligenes faecalis cellulitis after a dog bite case report and literature review. Pediatr Emer Care. 2017;33:497-8.

21. Ergul AB, Cetin $S$, Altintop YA, Bozdemir SE, Ozcan A, Altug U, et al. Evaluation of microorganisms causing ventilator-associated pneumonia in a pediatric intensive care unit. Eurasian J Med. 2017;49:87-91.

22. Agarwal A, Sharma S, Vivek B, Vibha B, Agarwal M, Airun M. First reported case of Alcaligenes faecalis isolated from bronchoalveolar lavage in a patient with dengue hemorrhagic fever. J Assoc Chest Physicians. 2017;5:51-5.

23. Junejo SZ, Tuli S, Trandafirescu T. A rare case of pneumonia caused by Alcaligenes faecalis bacteria. American Journal of Respiratory and Critical Care Medicine. 2018:197:A3602.

24. Chua KH, Puah SM, Puthucheary SD. First report of TEM-116 and OXA10 extended-spectrum $\beta$-lactamase in clinical isolates of Alcaligenes species from Kuala Lumpur, Malaysia. Jpn J Infect Dis. 2019;72:266-9.

25. Vogiatzis I, Koutsampasopoulos K. Infective endocarditis caused by Alcaligenes faecalis complicated with skin lesions. Anatol J Cardiol. 2019;21: 5010-1. https://doi.org/10.14744/AnatolJCardiol.2019.10576.

26. Hasan MJ, Nizhu LN, Rabbani R. Bloodstream infection with pandrugresistant Alcaligenes faecalis treated with double-dose of tigecycline. ID Cases. 2019;18:e00600. https://doi.org/10.1016/j.idcr.2019.e00600.

27. Al-Zakhari R, Suhail M, Ataallah B, Aljammali S, Grigos A. Rare but fetal case of cavitary pneumonia caused by Alcaligenes Faecalis. Cureus. 2020;12(6):e8934. https:/doi.org/10.7759/cureus.8934.

28. Majewski P, Majewska P, Gutowska A, Piszcz J, Sacha P, Wieczorek P, et al. Molecular characterisation of clinical pandrug-resistant Alcaligenes faecalis strain MUB14. Int J Antimicrob Agents. 2020;55:105939. https://doi.org/10. 1016/j.jijantimicag.2020.105939.

29. Huang C. Diabetic Foot Ulcer with Alcaligenes faecalis Infection. Dubai Diabetes Endocrinol J. 2020. https://doi.org/10.1159/000508094.

30. Magiorakosa AP, Srinivasann A, Carey RB, Carmelic Y, Falagasde ME, Giske $C G$, et al. Multidrug-resistant, extensively drug-resistant and pandrugresistant bacteria: an international expert proposal for interim standard definitions for acquired resistance. Clin Microbiol Infect. 2012;18(3):268-81. 
31. Terry LL, Mcbane JK, Dean KF. Bacteremia and acute meningitis due to Alcaligenes faecalis; a case report. J Lab Clin Med. 1947;32:1262-5.

32. Cole ACE, Marshall C. Endocarditis Due to Bact. Faecalis Alcaligenes. Br Med J. 1952;2:867. https://doi.org/10.1136/bmj.2.4789.867.

33. Warthen RO. Alcaligenes faecalis meningitis complicated by hydrocephalus in a premature twin; report of case; intramuscular chloramphenicol therapy. AMA Am J Dis Child. 1952;84:729-31. https://doi.org/10.1001/archpedi.1952. 02050060067005.

34. Berergo A, Bussinello E. An unusual case of subacute bacterial endocarditis caused by Bacillus faecalis alcaligenes; combined penicillin-sulfonamide therapy. Minerva Med. 1953;44:1322-7.

35. Dureux J, De LE, Burdin JC. A case of Alcaligenes faecalis septicemia. Rev Med Nancy. 1953;78:304-10.

36. Bozzo A, Pino G. A case of mixed meningitis caused by Alcaligenes faecalis and Flavobacterium arborescens. Policlinico Prat. 1953;60:109-13.

37. Librach IM. Faecalis alkaligenes infections with a report of a case of septicaemia. Postgrad Med J. 1954;30:424-9.

38. Mengedoth MM, Aton JK, Berry A. A case of measles encephalitis complicated by Alcaligenes faecalis septicemia. J Pediatr. 1960;57:738-40. https://doi.org/10.1016/s0022-3476(60)80168-0.

39. Joseph DS, David I, Joseph W, VP EC. Alcaligenes faecalis infection in the newborn. Am J Dis Child. 1960;100:212-6.

40. Doxiadis SA, Pavlatou M, Chryssostomidou O. Bacillus faecalis alcaligenes septicemia in the newborn. J Pediatr. 1960;56:648-54. https://doi.org/10. 1016/s0022-3476(60)80339-3.

41. Khetarpal SK. Alcaligenes faecalis septicemia in the newborn. Clin Pediatr (Phila). 1964;3:108-10. https://doi.org/10.1177/000992286400300211.

42. Bolletti M, Andrioli G. Acute meningitis due to Alcaligenes faecalis. (clinical contribution of 8 cases). G Mal Infett Parassit. 1967;19:353-9.

43. Berry AM. Alcaligenes faecalis septicemia and meningitis in the newborn. Report of an unusual case. Indian J Pediatr. 1967;34:242-4.

44. lelasi G, Calonghi GF, Sueri L. A case of progressive ascending purulent epiduritis caused by Alcaligenes faecalis. Infett Parassit. 1968;20:1027-9.

45. Codeceira A Jr, de Melo AB. Meningitis caused by Alcaligenes faecalis in adults. Report of a case. J Bras Psiquiatr. 1970;19:117-22.

46. Tayeri T, Kelly LD. Alcaligenes faecalis corneal ulcer in a patient with cicatricial pemphigoid. Am J Ophthalmol. 1993;115:255-6. https://doi.org/10. 1016/s0002-9394(14)73935-2.

47. Laham NA, Chavda KD, Cienfuegos-Gallet AV, Kreiswirth BN, Chen L. Genomic characterization of VIM Metallo- $\beta$-lactamase-producing Alcaligenes faecalis from Gaza, Palestine. Antimicrob Agents Chemother. 2017;61(11):e01499-17. https://doi.org/10.1128/AAC.01499-17.

48. Khajuria A, Praharaj AK, Kumar M, Grover N. Emergence of VIM-6 metallobeta-lactamase-producing Alcaligenes faecalis clinical isolates in a hospital in India. J Infect Dev Ctries. 2013;7:494-6. https://doi.org/10.3855/jidc.3556.

49. Ngbede EO, Anil P, Anwar K, Yang Y, Folasade A, Adikwu AA, et al. Identification of mobile colistin resistance genes (mcr-1.1, mcr-5, mcr-8.1) in Enterobacteriaceae and Alcaligenes faecalis of human and animal origins, Nigeria. Int J Antimicrob Agents. 2020;106108. https://doi.org/10.1016/j. ijantimicag.2020.106108.

50. Kim S, Kim MH, Lee WI, Kang SY, Jeon YL. Misidentification of Acinetobacter baumannii as Alcaligenes faecalis by VITEK 2 system. Case Report Lab Med. 2017;49:e14-7. https://doi.org/10.1093/labmed/Im×062.

51. Guo L, Ye L, Zhao Q, Ma Y, Yang J, Luo Y. Comparative study of MALDI-TOF MS and VITEK 2 in bacteria identification. J Thorac Dis. 2014;6:534-8. https:// doi.org/10.3978/j.issn.2072-1439.2014.02.18.

\section{Publisher's Note}

Springer Nature remains neutral with regard to jurisdictional claims in published maps and institutional affiliations.

\section{Ready to submit your research? Choose BMC and benefit from:}

- fast, convenient online submission

- thorough peer review by experienced researchers in your field

- rapid publication on acceptance

- support for research data, including large and complex data types

- gold Open Access which fosters wider collaboration and increased citations

- maximum visibility for your research: over $100 \mathrm{M}$ website views per year

At BMC, research is always in progress.

Learn more biomedcentral.com/submissions 